\title{
About Lorentz Invariance and Gauge Symmetries: An Alternative Approach to Relativistic Gravitation
}

\author{
Richard Bonneville \\ Centre National d'Etudes Spatiales (CNES), 2 Place Maurice Quentin, Paris, France \\ Email: richard.bonneville@cnes.fr
}

Received 11 September 2014; revised 26 September 2014; accepted 8 October 2014

Copyright (C) 2014 by author and Scientific Research Publishing Inc.

This work is licensed under the Creative Commons Attribution International License (CC BY).

http://creativecommons.org/licenses/by/4.0/

C) (i) Open Access

\begin{abstract}
An alternative presentation of a relativistic theory of gravitation, equivalent to general relativity, is given. It is based upon the restriction of the Lorentz invariance of special relativity from a global invariance to a local one. The resulting expressions appear rather simple as we consider the transformations of a local set of pseudo-orthonormal coordinates and not the geometry of a 4dimension hyper-surface described by a set of curvilinear coordinates. This is the major difference with the usual presentations of general relativity but that difference is purely formal. The usual approach is most adequate for describing the universe on a large scale in astrophysics and cosmology. The approach of this paper, derived from particle physics and focused on local reference frames, underlines the formal similarity between gravitation and the other interactions inasmuch as they are associated to the restriction of gauge symmetries from a global invariance to a local one.
\end{abstract}

\section{Keywords}

Gravitation, General Relativity, Gauge Symmetries

\section{Introduction}

In the usual presentations of general relativity [1], space-time geometry is described via any set of 4 curvilinear coordinates $\left\{q^{\alpha}\right\}$; an elementary space-time path $\mathrm{d} s$ is expressed as $\mathrm{d} s^{2}=-g_{\alpha \beta} \mathrm{d} q^{\alpha} \mathrm{d} q^{\beta}$ where the quadratic form $g_{\alpha \beta}$ characterizes the local metrics. The stake is to express the laws of physics in that frame; for example in the absence of other external forces than gravitation, a particle follows the minimum path $\int_{A}^{B} d s$ 
between two space-time points A and B. In the presence of a gravitation field there is no global transformation of the coordinates allowing the $g_{\alpha \beta}$ to take in the whole space-time the simple form of a Minkowski metrics; nevertheless it can take this special form in a particular point by a suitable change of coordinates which simply consists in diagonalizing $g_{\alpha \beta}$ in this point [2].

We give here an alternative presentation of a relativistic theory of gravitation, equivalent to general relativity, in which gravitation is introduced as a gauge field associated to restricting the global Lorentz invariance of special relativity to a local one.

The presentation of gravitation as a gauge field has been first introduced by Lanczos in the context of a variational approach to general relativity [3] [4]. Then it has been highlighted in an extension of the Yang-Mills approach of particle physics [5]-[7]. In the later, the fundamental interactions are accounted for by the restriction of gauge symmetries from a global invariance to a local one [8]. Those interactions (electro-magnetic, weak, strong) are associated to symmetry groups isomorphic to $\mathrm{U}(1), \mathrm{SU}(2), \mathrm{SU}(3)$, whose elements are unitary transformations [9] [N.B.: by isomorphism between two groups, we mean that their Lie algebras possess the same commutation relations]. When that approach is applied to gravitation, the equivalence principle, central in general relativity [10], is not explicitly assumed but rather derived from the fact that the gauge field is supposed to be minimally coupled.

That restriction from a global invariance to a local one, associated to the emergence of a force field, is indeed a deep similarity between gravitation and the other interactions. However the Lorentz group is isomorphic to the special complex linear group SL(2,C) whose elements are not unitary and this is an essential difference between gravitation and the other interactions.

In the present work, we assume that the Lorentz group invariance is not a global symmetry of space-time but a local symmetry of a 4-dimension hyper-surface, which can be thought of as embedded in a space with a larger number of dimensions and we consider the transformations of a local set of pseudo-orthonormal coordinates and not the geometry of the 4-dimension hyper-surface described by a set of curvilinear coordinates. This is a major difference with other presentations of relativistic gravitation; its interest is to lead to facially simpler expressions although that difference is essentially formal.

\section{Gauge Properties}

Let us consider the 4-dimension space-time of special relativity and a pseudo-orthonormal base $\left\{\mathbf{e}_{0}, \mathbf{e}_{1}, \mathbf{e}_{2}, \mathbf{e}_{3}\right\}$ with the Minkowski metrics

$$
\eta_{\mu v}=\left(\begin{array}{cccc}
-1 & 0 & 0 & 0 \\
0 & 1 & 0 & 0 \\
0 & 0 & 1 & 0 \\
0 & 0 & 0 & 1
\end{array}\right)
$$

Any infinitesimal transformation $\mathbf{R}$ of the Lorentz group can be written as

$$
\mathbf{R}=\mathbf{I}+\left(\begin{array}{cccc}
0 & -\beta_{x} & -\beta_{y} & -\beta_{z} \\
-\beta_{x} & 0 & \alpha_{z} & -\alpha_{y} \\
-\beta_{y} & -\alpha_{z} & 0 & \alpha_{x} \\
-\beta_{z} & \alpha_{y} & -\alpha_{x} & 0
\end{array}\right)
$$

$\mathbf{R}$ conserves the pseudo norm $-x_{0}^{2}+x_{1}^{2}+x_{2}^{2}+x_{3}^{2}=-c^{2} t^{2}+x^{2}+y^{2}+z^{2}$. It can alternatively be written as

$$
\mathbf{R}=\mathbf{I}+i \overrightarrow{\boldsymbol{\alpha}} \cdot \overrightarrow{\mathbf{J}}+i \overrightarrow{\boldsymbol{\beta}} \cdot \overrightarrow{\mathbf{K}}
$$

where $\overrightarrow{\boldsymbol{\alpha}}$ et $\overrightarrow{\boldsymbol{\beta}}$ are 2 vectors of the ordinary 3-dimension-space. $\mathbf{J}_{x}, \mathbf{J}_{y}, \mathbf{J}_{z}, \mathbf{K}_{x}, \mathbf{K}_{y}, \mathbf{K}_{z}$ are the 6 infinitesimal generators of the Lorentz group $\Lambda\left(\underset{\vec{J}}{)}\right.$, the $\mathbf{J}_{p}$ s are hermitic $\left(\mathbf{J}_{p}^{\dagger}=\mathbf{J}_{p}\right)$, whereas the $\mathbf{K}_{p} \mathrm{~s}$ are antihermitic $\left(\mathbf{K}_{p}^{\dagger}=-\mathbf{K}_{p}\right)$, the pseudo vector $\overrightarrow{\mathbf{J}}$ and the true vector $\overrightarrow{\mathbf{K}}$ respectively account for rotations and for Lorentz transformations; $\alpha_{z}$ stands for a rotation angle around the $\mathrm{Z}$ axis, and $\beta_{z}$ for the velocity of a Lorentz transformation along the $\mathrm{Z}$ axis.

We introduce the 2 anti-symmetric tensors 


$$
\mathbf{L}^{\rho \sigma}=\left(\begin{array}{cccc}
\mathbf{0} & \mathbf{K}_{x} & \mathbf{K}_{y} & \mathbf{K}_{z} \\
-\mathbf{K}_{x} & \mathbf{0} & \mathbf{J}_{z} & -\mathbf{J}_{y} \\
-\mathbf{K}_{y} & -\mathbf{J}_{z} & \mathbf{0} & \mathbf{J}_{x} \\
-\mathbf{K}_{z} & \mathbf{J}_{y} & -\mathbf{J}_{x} & \mathbf{0}
\end{array}\right)
$$

and

$$
\boldsymbol{\Omega}_{\rho \sigma}=\left(\begin{array}{cccc}
0 & \beta_{x} & \beta_{y} & \beta_{z} \\
-\beta_{x} & 0 & \alpha_{z} & -\alpha_{y} \\
-\beta_{y} & -\alpha_{z} & 0 & \alpha_{x} \\
-\beta_{z} & \alpha_{y} & -\alpha_{x} & 0
\end{array}\right)
$$

Each component $\mathbf{J}_{p}$ or $\mathbf{K}_{p}$ is a $4 \times 4$ matrix so that $\mathbf{R}$ can be written as

$$
\mathbf{R}=\mathbf{I}+\frac{i}{2} \underline{\underline{\boldsymbol{\Omega}}}: \underline{\underline{\mathbf{L}}}=\mathbf{I}+\frac{i}{2} \boldsymbol{\Omega}_{\rho \sigma} \mathbf{L}^{\rho \sigma}
$$

More generally, any transformation of the Lorentz group can be figured by

$$
\mathbf{R}(\boldsymbol{\Omega})=\exp \frac{i}{2} \underline{\underline{\Omega}}: \underline{\mathbf{L}}
$$

We now assume that the Lorentz group invariance is not a global symmetry of space-time but a local symmetry of a 4-dimension hyper-surface, which can be thought of as embedded in a space with a larger number of dimensions, for example 10 as it is envisaged in many unification theories. If we consider the tangent plane to this hyper-surface in any point $M$, it is possible to define in this plane a pseudo-orthonormal reference frame, and in fact an infinite set of similar frames deduced from each other by a Lorentz transformation or a rotation; in the close vicinity of $M$ the laws of physics are invariant under the Lorentz group. We can in another point $M^{\prime}$ of the hyper-surface $M^{\prime}$ define a similar set of pseudo-orthonormal frames. The question is: what is the correspondence between the 2 sets of reference frames attached to $M$ and $M^{\prime}$ ?

Let us first perform on the surface an infinitesimal displacement $\mathbf{T}(\boldsymbol{\varepsilon})$ from $M$ to $M^{\prime}=M+\mathrm{d} M$; in this close vicinity of $M$ the surface is assimilated to its tangent plane and $\mathbf{T}(\boldsymbol{\varepsilon})$ is an infinitesimal translation. Then we perform a transformation $\mathbf{R}(\boldsymbol{\Omega})$ around $M^{\prime}$. If $\boldsymbol{\Omega}$ is a constant, the coordinates of $M^{\prime}$ are

$$
x^{\prime \mu}=\left[\exp \frac{i}{2} \underline{\underline{\boldsymbol{\Omega}}}: \underline{\underline{\mathbf{L}}}\right]_{v}^{\mu}\left(x^{v}+\varepsilon^{v}\right)=\left[\exp \frac{i}{2} \underline{\underline{\boldsymbol{\Omega}}}: \underline{\underline{\mathbf{L}}}\right]_{v}^{\mu} x^{v}+\left[\exp \frac{i}{2} \underline{\underline{\boldsymbol{\Omega}}}: \underline{\underline{\mathbf{L}}}\right]_{v}^{\mu} \varepsilon^{v}
$$

But if $\Omega$ is a function of the point $M$, that expression becomes:

$$
\begin{aligned}
x^{\prime \mu} & =\left[\exp \frac{i}{2} \underline{\underline{\boldsymbol{\Omega}}}(M+\mathrm{d} M): \underline{\underline{\mathbf{L}}}\right]_{v}^{\mu}\left(x^{v}+\varepsilon^{v}\right) \\
& =\left[\exp \frac{i}{2} \underline{\underline{\boldsymbol{\Omega}}}(M): \underline{\underline{\mathbf{L}}}\right]_{v}^{\mu} x^{v}+\left[\exp \frac{i}{2} \underline{\underline{\boldsymbol{\Omega}}}: \underline{\underline{\mathbf{L}}}\right]_{v}^{\mu} \varepsilon^{v}+\frac{i}{2}\left[\exp \frac{i}{2} \underline{\underline{\boldsymbol{\Omega}}: \underline{\underline{\mathbf{L}}}]\left[\varepsilon^{\rho} \partial_{\rho} \underline{\underline{\boldsymbol{\Omega}}}: \underline{\underline{\mathbf{L}}}\right]_{v}^{\mu} x^{v}}\right.
\end{aligned}
$$

Comparing the two expressions Equation (8) and Equation (9) above, we see that $\varepsilon^{\mu}$ is transformed into

$$
\varepsilon^{\mu}+\varepsilon^{v} \boldsymbol{G}_{v}^{\mu}
$$

with

$$
\mathbf{G}_{v}^{\mu}=\frac{i}{2}\left[\partial_{\nu} \underline{\underline{\boldsymbol{\Omega}}}: \underline{\underline{\mathbf{L}}}\right]_{\rho}^{\mu} x^{\rho}
$$

Now considering some function $\Phi(M)$, we deduce that

$$
\boldsymbol{\Phi}(M+\mathrm{d} M)=\boldsymbol{\Phi}\left(x^{\mu}\right)+\varepsilon^{\mu} \partial_{\mu} \Phi
$$

is in a similar way transformed into 


$$
\boldsymbol{\Phi}\left(x^{\mu}\right)+\varepsilon^{\mu} \partial_{\mu} \boldsymbol{\Phi}+\varepsilon^{v} \mathbf{G}_{\nu}^{\mu} \partial_{\mu} \boldsymbol{\Phi}
$$

i.e. $\partial_{\mu}$ is replaced by

$$
D_{\mu}=\partial_{\mu}+\mathbf{G}_{\mu}^{v} \partial_{v}
$$

The impulsion

$$
\mathbf{p}^{\mu}=i^{-1} \partial^{\mu}=i^{-1} \boldsymbol{\eta}^{\mu v} \partial_{v}=\left(i \frac{\partial}{\partial t}, i^{-1} \vec{\nabla}\right)
$$

is the infinitesimal generator of space-time translations. Equation (14) above means that $\mathbf{p}^{\mu}$ is replaced by

$$
\mathbf{P}^{\mu}=i^{-1} D^{\mu}=\mathbf{p}^{\mu}+G_{\lambda}^{\mu} \mathbf{p}^{\lambda}
$$

The orbital angular momentum anti-symmetric tensor

$$
\mathbf{l}^{\rho \sigma}=\mathbf{x}^{\rho} \mathbf{p}^{\sigma}-\mathbf{x}^{\sigma} \mathbf{p}^{\rho}=i^{-1}\left(x^{\rho} \partial^{\sigma}-x^{\sigma} \partial^{\rho}\right)
$$

can be written as

$$
\mathbf{l}^{\rho \sigma}=i^{-1} \partial / \partial \varphi_{\rho \sigma}
$$

where $\varphi_{\rho \sigma}$ denotes a rotation angle in the $(\rho \sigma)$ plan (N.B.: the 3-dimension vector components $\mathbf{j}_{p}=\varepsilon_{p q r}{ }^{q r}$ and $\mathbf{k}_{p}=\mathbf{l}^{0 p}$ also satisfy the commutation relations above). Since $\boldsymbol{\Omega}_{\rho \sigma}=-\boldsymbol{\Omega}_{\sigma \rho}$, then

$$
D_{\mu}=\partial_{\mu}+\frac{i}{2} x^{\rho}\left[\partial_{\mu} \underline{\underline{\boldsymbol{\Omega}}}: \underline{\mathbf{L}}\right]_{\rho}^{v} \partial_{v}
$$

can be written as

$$
D_{\mu}=\partial_{\mu}+\frac{i}{4} \boldsymbol{\eta}_{\mu \sigma}\left[\frac{\partial \underline{\underline{\underline{\Omega}}}}{\partial \varphi_{\rho \sigma}}: \underline{\underline{\mathbf{L}}}\right]_{\rho}^{v} \partial_{\nu}
$$

That expression allows to evidence a gauge invariance property: it is possible to add to $\boldsymbol{\Omega}_{\rho \sigma}$ any function $\omega\left(\eta_{\mu v} x^{\mu} x^{v}\right)$ without changing $\mathbf{G}_{v}^{\mu}$ : if $\omega$ only depends on the invariant quantity $\eta_{\mu v} x^{\mu} x^{\nu}$, its derivation with respect to $\varphi_{\rho \sigma}$ is just 0 . As a consequence, there is some flexibility in the determination of the $\mathbf{G}_{v}^{\mu} \mathrm{s}$ and we can impose the 4 gauge conditions

$$
\partial_{\mu} \mathbf{G}_{v}^{\mu}=0
$$

We have here above considered the transformations of a local set of pseudo-orthonormal coordinates and not the geometry of the 4-dimension hyper-surface described by a set of curvilinear coordinates. This is the major difference with other presentations of relativistic gravitation and notably with general relativity but that difference is purely formal. As a consequence, many mathematical expressions look simpler; for example, the invariant 4-dimension volume element $\mathrm{d} V$ that appears in the expression of the action integral $S=\int \mathcal{L} \mathrm{d} V$ in the Lagrange formalism is merely $\mathrm{d}^{4} \mathbf{x}$, i.e. the determinant of the metric tensor is 1 .

\section{Gravitation Field Equations}

We now consider a scalar particle of mass $m$ (but the procedure can be straightforwardly generalized to a particle of any spin, be it massive or not) and the Lagrangian density

$$
\mathcal{L}=\eta^{\mu v} \dot{\Psi}_{\mu}^{\dagger} \dot{\Psi}_{v}+(m c / \hbar)^{2} \Psi^{\dagger} \Psi
$$

with

$$
\dot{\Psi}_{\mu}=\partial_{\mu} \Psi
$$

We perform the transformation $\partial_{\mu} \rightarrow D_{\mu}=\partial_{\mu}+\mathbf{G}_{\mu}^{v} \partial_{v}$ so that the Lagrangian density becomes 


$$
\mathcal{L}=\mathfrak{g}^{\mu \nu} \dot{\Psi}_{\mu}^{\dagger} \dot{\Psi}_{v}+(m c / \hbar)^{2} \Psi^{\dagger} \Psi
$$

where we have introduced the effective metrics

$$
\mathfrak{g}_{\mu v}=\boldsymbol{\eta}_{\mu v}+\mathfrak{h}_{\mu v}
$$

with

$$
\mathfrak{h}_{\mu v}=\boldsymbol{\eta}_{\rho \sigma} \mathbf{G}_{\mu}^{\rho} \mathbf{G}_{v}^{\sigma}+\boldsymbol{\eta}_{\mu \rho} \mathbf{G}_{v}^{\rho}+\boldsymbol{\eta}_{v \sigma} \mathbf{G}_{\mu}^{\sigma}
$$

We have written $\mathfrak{g}_{\mu \nu}$ and $\mathfrak{h}_{\mu v}$ with gothic letters instead of the usual $\mathbf{g}_{\mu v}$ and $\mathbf{h}_{\mu v}$ in order to remind ourselves that we have considered a local set of pseudo Cartesian coordinates and not a set of curvilinear coordinates running over the whole surface.

Applying the Lagrange equations to the $\Psi$ field, i.e.

$$
\partial_{\mu} \frac{\partial \mathcal{L}}{\partial \dot{\Psi}_{\mu}}=\frac{\partial \mathcal{L}}{\partial \Psi}
$$

gives

$$
\left[\mathbf{g}^{\mu \nu} \partial_{\mu} \partial_{\nu}-(m c / \hbar)^{2}\right] \Psi+\left(\partial_{\mu} \mathbf{q}^{\mu \nu}\right)\left(\partial_{\nu} \Psi\right)=0
$$

From the expression of $\mathfrak{g}^{\mu \nu}$ as a function of the $\mathbf{G}_{\mu \nu}$ s it is clear that $\mathfrak{g}^{\mu \nu}=\mathfrak{g}^{v \mu} ; \mathfrak{g}$ has thus 10 components but from the gauge conditions $\partial_{\mu} \mathbf{G}_{v}^{\mu}=0$ we derive

$$
\partial_{\mu} \mathbf{g}^{\mu v}=0
$$

Equation (26) shows that actually has only 6 independent components. The cross-term in Equation (25) vanishes, leading to

$$
\left(\mathbf{g}^{\mu v} \partial_{\mu} \partial_{v}\right) \Psi-(m c / \hbar)^{2} \Psi=0
$$

The wave equation so appears as the wave equation of a free particle in which the original Minkowski metrics $\eta_{\mu v}$ has been replaced by the effective metrics $\mathfrak{g}_{\mu v}$.

The gravitation field is thus described by a modification of the geometry of space-time by replacing the Minkowski metrics $\eta_{\mu v}$ by the effective metrics $\mathfrak{g}_{\mu v}$. A consequence of the modified wave equation above is that the dynamics of any particle, with or without mass, is affected by a gravitation field.

We now assume for the gravitation field itself a Lagrangian density term quadratic in $\mathbf{g}$ and in $\partial_{\mu} \mathbf{g}$. The scalar product of two vectors $\mathbf{A}$ and $\mathbf{B}$ is $\mathbf{g}_{\mu v} A^{\mu} B^{v}=A_{\mu} B^{\mu}=A^{\mu} B_{\mu}$, this can be extended to the scalar product of two tensors of any rank and to the metric tensor itself. So for the term quadratic in $\mathbf{g}$ we assume the expression

$$
\lambda^{2} \mathbf{q}_{\alpha \rho} \mathbf{g}_{\beta \sigma} \mathbf{g}^{\rho \sigma} \mathbf{g}^{\alpha \beta}=\lambda^{2} \mathbf{g}_{\sigma}^{\alpha} \mathbf{q}_{\alpha}^{\sigma}=\lambda^{2} \mathbf{g}^{\alpha \beta} \mathbf{g}_{\alpha \beta}
$$

$\lambda$ is the mass of the gravitation field; now $\mathbf{g}^{\alpha \beta} \mathbf{g}_{\alpha \beta}=\mathbf{I}$ so that this term is a constant we can hereunder discard (in fact we will see later on that formally maintaining that term in the calculation would imply $\lambda=0$ ). In a similar way we assume for the term quadratic in $\partial_{\mu} \mathbf{g}$ the following expression

$$
\mathbf{q}^{\mu v}\left(\mathfrak{g}_{\alpha \rho} \partial_{\mu} \mathfrak{g}^{\rho \beta}\right)\left(\mathfrak{q}_{\beta \sigma} \partial_{\nu} \mathbf{g}^{\sigma \alpha}\right)
$$

or

$$
\left(\partial^{v} \mathbf{g}_{\rho \sigma}\right)\left(\partial_{\nu} \mathfrak{g}^{\rho \sigma}\right)
$$

[N.B.: as $\mathfrak{g}^{\mu v} \mathbf{g}_{\mu v}^{\mu v}=\mathbf{I}$, considering the infinitesimal variations $\mathfrak{g}^{\mu v} \rightarrow \mathfrak{g}^{\mu v}+\boldsymbol{\delta}^{\mu v}$ and $\mathfrak{g}_{\mu v} \rightarrow \mathfrak{g}_{\mu v}+\boldsymbol{\delta}_{\mu v}$, and neglecting the second order terms gives $\delta^{\mu \nu}=-\delta_{\mu v}$; it then may be checked that the additional terms that could appear in developing the derivatives of Equation (29b) actually cancel each other].

The full Lagrangian density of the (field + particle) system is

$$
\mathcal{L}=\left[\mathbf{g}^{\mu v} \partial_{\mu} \Psi^{\dagger} \partial_{\nu} \Psi+(m c / \hbar)^{2} \Psi^{\dagger} \Psi\right]-\chi^{-1} \mathbf{g}^{\mu v}\left(\mathbf{g}_{\alpha \rho} \partial_{\mu} \mathbf{g}^{\rho \beta}\right)\left(\mathbf{g}_{\beta \sigma} \partial_{\nu} \mathfrak{g}^{\sigma \alpha}\right)
$$


where $\chi$ is a dimensionless constant; the negative sign and the negative exponent are for commodity reasons.

Applying the Lagrange equations to $\mathfrak{g}^{\mu \nu}$, i.e.

$$
\partial_{\xi} \frac{\partial \mathcal{L}}{\partial \dot{\mathbf{q}}^{\mu \nu}}=\frac{\partial \mathcal{L}}{\partial \mathbf{q}^{\mu \nu}}
$$

with $\dot{\mathfrak{g}}^{\mu v}=\partial_{\xi} \mathfrak{g}^{\mu v}$ leads to the field equations (with the same remarks as in the N.B. here above):

$$
\mathfrak{g}_{\mu \alpha} \mathfrak{g}_{\nu \beta}\left(\mathfrak{g}^{\rho \sigma} \partial_{\rho} \partial_{\sigma} \mathfrak{g}^{\alpha \beta}\right)=-\chi\left(\partial_{\mu} \Psi\right)^{\dagger}\left(\partial_{\nu} \Psi\right)
$$

These are the equations of the gravitation field and their nonlinear character is obvious. The term on the righthand side

$$
\chi\left(\partial_{\mu} \Psi\right)^{\dagger}\left(\partial_{\nu} \Psi\right)
$$

is proportional to the energy-impulsion density tensor of the particle; it is the source term of the gravitation field. In the classical, i.e. non quantum, limit, the correspondence $\mathbf{p}_{\mu} \leftrightarrow i^{-1} \hbar \boldsymbol{\eta}_{\mu}^{v} \partial_{v}$ together with the expression of the density $\rho=m \Psi^{\dagger} \Psi$, changes this term into

$$
\chi \frac{\rho}{m \hbar^{2}} \mathbf{p}_{\mu} \mathbf{p}_{v}=\chi \frac{\rho}{m \hbar^{2}} \mathbf{T}_{\mu v}
$$

So Equation (32) becomes

$$
\mathbf{g}^{\mu v} \mathbf{g}_{\mu \alpha} \mathbf{q}_{\nu \beta}\left(\mathbf{g}^{\rho \sigma} \partial_{\rho} \partial_{\sigma} \mathbf{g}^{\alpha \beta}\right)=-\chi \frac{\rho}{m \hbar^{2}} \mathfrak{y}^{\mu v} \mathbf{T}_{\mu v}
$$

For the sake of commodity, we will re-write it in a different, more workable way. Let us introduce the two quantities

$$
\begin{gathered}
\mathrm{T}=\mathfrak{g}^{\mu \nu} \mathbf{T}_{\mu \nu} \\
\boldsymbol{\tau}_{\mu \nu}=\mathbf{T}_{\mu v}-\frac{1}{2} \mathfrak{g}_{\mu v} \mathrm{~T}
\end{gathered}
$$

Combining Equations (35) and (36) we finally get after some manipulations

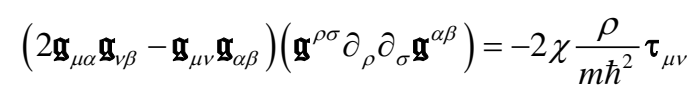

\section{Non Relativistic Limit}

In the case of a weak gravitation field, the quadratic terms in the field equations can be neglected. In the absence of matter, the linearized equations take the form of propagation like equations:

$$
\eta^{\mu v} \partial_{\mu} \partial_{\nu} \mathfrak{h}_{\mu \nu} \approx 0
$$

If matter is present, there is a source term:

$$
\left(2 \boldsymbol{\eta}_{\mu \alpha} \boldsymbol{\eta}_{\nu \beta}-\boldsymbol{\eta}_{\mu \nu} \boldsymbol{\eta}_{\alpha \beta}\right)\left(\boldsymbol{\eta}^{\rho \sigma} \partial_{\rho} \partial_{\sigma} \mathfrak{h}^{\alpha \beta}\right) \approx-2 \chi \frac{\rho}{m \hbar^{2}}\left(\mathbf{T}_{\mu \nu}-\frac{1}{2} \boldsymbol{\eta}_{\mu v} \mathrm{~T}\right)
$$

with

$$
\mathrm{T} \approx \boldsymbol{\eta}^{\rho \sigma} \mathbf{T}_{\rho \sigma}
$$

i.e.

$$
\begin{aligned}
\square \mathfrak{h}_{00} & \approx-2 \chi \frac{\rho}{m \hbar^{2}}\left(\mathbf{T}_{00}+\frac{\mathrm{T}}{2}\right) \\
\square \mathfrak{h}_{i i} & \approx-2 \chi \frac{\rho}{m \hbar^{2}}\left(\mathbf{T}_{i i}-\frac{\mathrm{T}}{2}\right)
\end{aligned}
$$




$$
\begin{aligned}
\square \mathfrak{h}_{i 0} & \approx+\chi \frac{\rho}{m \hbar^{2}} \mathbf{T}_{i 0} \\
\square \mathfrak{h}_{i \neq j} & \approx-\chi \frac{\rho}{m \hbar^{2}} \mathbf{T}_{i j}
\end{aligned}
$$

In the non-relativistic limit $\mathbf{E} \approx m c^{2} \gg \mathbf{p} c \gg \mathbf{p}^{2} c^{2} / m c^{2}$ and hence we get

$$
\begin{aligned}
& \mathbf{T}_{00} \approx m^{2} c^{4} \\
& \mathbf{T}_{i i} \approx 0 \\
& \mathbf{T}_{i 0} \approx 0 \\
& \mathbf{T}_{i \neq j} \approx 0
\end{aligned}
$$

hence

$$
\mathrm{T} \approx-m^{2} c^{4}
$$

Moreover if the field is slowly varying with time, the time derivatives on the left hand side of Equation (40) vanish and those equations become:

$$
\begin{gathered}
\nabla^{2} \mathfrak{h}_{00} \approx-\chi \frac{\rho}{\hbar^{2}} m c^{2} \\
\nabla^{2} \mathfrak{h}_{i i} \approx-\chi \frac{\rho}{\hbar^{2}} m c^{2} \\
\nabla^{2} \mathfrak{h}_{i 0} \approx 0 \\
\nabla^{2} \mathfrak{h}_{i \neq j} \approx 0
\end{gathered}
$$

The above expression for $\mathfrak{b}_{00}$ has to be compared with the expression of the classical gravitation potential $\mathbf{U}$ :

$$
\nabla^{2} \mathbf{U}=4 \pi \rho G
$$

where $G$ is the gravitation constant. It means that in the non-relativistic limit $\mathfrak{b}_{00}$ is proportional to the Newtonian potential. The constant $\chi$ will be hereunder determined by the requirement of compatibility with Newton's equation of motion.

On the other hand, the dynamical equation of a massive particle is given by Equation (27) $\left(\mathbf{g}^{\mu v} \partial_{\mu} \partial_{v}\right) \Psi-(m c / \hbar)^{2} \Psi=0$ which in the classical limit becomes

$$
\mathbf{g}^{\mu v} \mathbf{p}_{\mu} \mathbf{p}_{v}=-m^{2} c^{2}
$$

or

$$
\mathbf{g}^{00} \mathbf{E}^{2}+\sum_{i=1,2,3} \mathbf{g}^{0 i} \mathbf{E} \mathbf{p}_{i} c+\sum_{i=1,2,3} \mathfrak{g}^{i i} \mathbf{p}_{i}^{2} c^{2}+\sum_{i \neq j} \mathbf{g}^{i j} \mathbf{p}_{i} \mathbf{p}_{j} c^{2}=-m^{2} c^{4}
$$

Let us put

$$
\mathfrak{g}^{\mu v}=\boldsymbol{\eta}^{\mu v}+\mathbf{t}^{\mu v}
$$

so that Equation (44b) becomes

$$
\left(-1+\mathbf{f}^{00}\right) \mathbf{E}^{2}+\sum_{i=1,2,3} \mathfrak{f}^{0 i} \mathbf{E} \mathbf{p}_{i} c+\sum_{i=1,2,3}\left(1+\mathbf{f}^{i i}\right) \mathbf{p}_{i}^{2} c^{2}+\sum_{i \neq j} \mathbf{f}^{i j} \mathbf{p}_{i} \mathbf{p}_{j} c^{2}=-m^{2} c^{4}
$$

or

$$
\mathbf{E}^{2}=\left(m^{2} c^{4}+\mathbf{p}^{2} c^{2}\right)+\mathfrak{f}^{00} \mathbf{E}^{2}+\sum_{i=1,2,3} \mathbf{f}^{i i} \mathbf{p}_{i}{ }^{2} c^{2}+\sum_{i=1,2,3} \mathfrak{f}^{0 i} \mathbf{E}_{i} c+\sum_{i \neq j} \mathbf{f}^{i j} \mathbf{p}_{i} \mathbf{p}_{j} c^{2}
$$

Let us also put 


$$
\mathbf{E}_{(0)}=\left(m^{2} c^{4}+\mathbf{p}^{2} c^{2}\right)^{1 / 2}
$$

We proceed by successive iterations; replacing $\mathbf{E}$ by $\mathbf{E}_{(0)}$ in the right hand side of Equation (46b) gives

$$
\mathbf{E}_{(1)}^{2} \approx \mathbf{E}_{(0)}^{2}+\mathfrak{f}^{00} \mathbf{E}_{(0)}^{2}+\sum_{i=1,2,3} \mathfrak{f}^{i i} \mathbf{p}_{i}^{2} c^{2}+\sum_{i=1,2,3} \mathbf{f}^{0 i} \mathbf{E}_{(0)} \mathbf{p}_{i} c+\sum_{i \neq j} \mathfrak{t}^{i j} \mathbf{p}_{i} \mathbf{p}_{j} c^{2}
$$

or

$$
\mathbf{E}_{(1)}^{2} \approx \mathbf{E}_{(0)}^{2}+\mathbf{f}^{00} m^{2} c^{4}+\sum_{i=1,2,3}\left(\mathbf{f}^{00}+\mathbf{f}^{i i}\right) \mathbf{p}_{i}^{2} c^{2}+\sum_{i=1,2,3} \mathbf{f}^{0 i} m c^{2} \mathbf{p}_{i} c+\sum_{i \neq j} \mathbf{f}^{i j} \mathbf{p}_{i} \mathbf{p}_{j} c^{2}
$$

In the non-relativistic limit $\mathbf{E} \approx m c^{2} \gg \mathbf{p} c \gg \mathbf{p}^{2} c^{2} / m c^{2}$ so that Equation (48b) approximately becomes

$$
\mathbf{E}_{(1)}^{2} \approx \mathbf{E}_{(0)}^{2}+\mathbf{f}^{00} m^{2} c^{4}
$$

Moreover in the weak field limit $\left|\mathfrak{f}^{00}\right| \ll 1$ so that from Equation (49) we finally derive

$$
\mathbf{E}_{(0)} \approx m c^{2}+\frac{\mathbf{p}^{2}}{2 m}+\frac{\mathbf{f}^{00} m c^{2}}{2}
$$

If we compare the above expression with the non-relativistic expression

$$
\mathbf{E}=m c^{2}+\frac{\mathbf{p}^{2}}{2 m}+m \mathbf{U}
$$

we see that the particle is undergoing an effective gravitation potential

$$
\mathbf{U}=\mathbf{f}^{00} c^{2} / 2
$$

Since $\mathfrak{g}^{\mu v} \mathbf{g}_{\mu v}=\mathbf{I}$ this is equivalent to

$$
\mathbf{U}=-\mathfrak{h}_{00} c^{2} / 2
$$

Since from Equation (42a) $\nabla^{2} \mathfrak{b}_{00} \approx-\chi \frac{\rho}{\hbar^{2}} m c^{2}$ the above expression of $\mathbf{U}$ yields

$$
\nabla^{2} \mathbf{U} \approx \chi \frac{\rho m c^{4}}{2 \hbar^{2}}
$$

By comparison with the expression of the classical gravitation potential $\nabla^{2} \mathbf{U}=4 \pi \rho G$ we get

$$
\chi=\frac{8 \pi G \hbar^{2}}{m c^{4}}
$$

If we had retained the $\lambda^{2} \mathbf{s}^{\alpha \beta} \mathbf{g}_{\alpha \beta}$ term in the expression Equation (30) of the Lagrangian density of the gravitation field, we would have in Equation (52b) an additional constant term; the comparison with the classical gravitation potential implies $\lambda=0$. The field equation Equation (37) now writes as

$$
\left(2 \mathbf{g}_{\mu \alpha} \mathbf{g}_{v \beta}-\mathfrak{g}_{\mu \nu} \mathbf{g}_{\alpha \beta}\right)\left(\mathbf{g}^{\rho \sigma} \partial_{\rho} \partial_{\sigma} \mathbf{g}^{\alpha \beta}\right)=-\frac{16 \pi \rho G}{m^{2} c^{4}} \boldsymbol{\tau}_{\mu \nu}
$$

\section{Post-Newtonian Terms}

From the expressions Equation (42) we derive in the weak, slowly varying field limit

$$
\begin{gathered}
\mathfrak{g}_{00} \approx-1-2 \frac{\mathbf{U}}{c^{2}} \\
\mathfrak{g}_{i i} \approx 1-2 \frac{\mathbf{U}}{c^{2}} \\
\mathfrak{g}_{0 i} \approx 0 \\
\mathfrak{g}_{i \neq j} \approx 0
\end{gathered}
$$


so that

$$
\mathrm{d} s^{2} \approx-\left(1+2 \frac{\mathbf{U}}{c^{2}}\right) c^{2} \mathrm{~d} t^{2}+\left(1-2 \frac{\mathbf{U}}{c^{2}}\right)\left(\mathrm{d} x^{2}+\mathrm{d} y^{2}+\mathrm{d} z^{2}\right)
$$

In addition, Equation (48b) gives

$$
\mathbf{E}_{(1)}^{2} \approx \mathbf{E}_{(0)}^{2}+2 m^{2} c^{2} \mathbf{U}+4 \frac{\mathbf{U}}{c^{2}} \mathbf{p}^{2} c^{2}
$$

This has to be compared with the Newtonian expression at the lowest order in $\mathbf{p}^{2} c^{2}$ and $m \mathbf{U}$

$$
\mathbf{E}^{2}=m^{2} c^{4}+\mathbf{p}^{2} c^{2}+2 m^{2} c^{2} \mathbf{U}+\frac{\mathbf{U}}{c^{2}} \mathbf{p}^{2} c^{2}+o\left(m^{2} \mathbf{U}^{2}, \mathbf{p}^{4} c^{4}\right)
$$

or

$$
\mathbf{E}^{2} \approx \mathbf{E}_{(0)}^{2}+2 m^{2} c^{2} \mathbf{U}+\frac{\mathbf{U}}{c^{2}} \mathbf{p}^{2} c^{2}
$$

\section{Conclusions}

Introducing the Planck length $L_{P}=\left(\hbar c^{-3} G\right)^{1 / 2}$ and the Planck mass $M_{P}=\left(\hbar c G^{-1}\right)^{1 / 2}$ leads to the following expression for the full Lagrangian density of the (field + particle) system

$$
\mathcal{L}=\left[\mathfrak{g}^{\rho \sigma} \partial_{\rho} \Psi^{\dagger} \partial_{\sigma} \Psi+(m c / \hbar)^{2} \Psi^{\dagger} \Psi\right]-\frac{1}{8 \pi} \frac{m}{M_{P}} \frac{1}{L_{P}^{3}}\left[\mathfrak{g}^{\mu v}\left(\mathfrak{g}_{\alpha \rho} \partial_{\mu} \mathbf{g}^{\rho \beta}\right)\left(\mathfrak{g}_{\beta \sigma} \partial_{v} \mathbf{g}^{\sigma \alpha}\right)\right]
$$

$\left(M_{P} \approx 2.17651 \times 10^{-8} \mathrm{~kg}\right)$. According to that expression, the physical objects such as $m \ll M_{P}$ have their dynamics mainly driven by the forces other than gravitation and the effective space-time metrics $\mathrm{g}$ is the metrics generated by the external masses. For the objects such as $m \gg M_{P}$, gravitation is a major driver of their dynamics, and they generate the gravitation field as well as they undergo it.

The usual approach of general relativity is most adequate for describing the universe on a large scale in astrophysics and cosmology. The approach of this paper, derived from particle physics and focused on local reference frames, underlines the formal similarity between gravitation and the other interactions inasmuch as they are associated to the restriction of a global symmetry to a local one.

In a 10-dimension space-time as it is considered in certain unification theories, gravitation is linked to the geometry of the 4 usual dimensions whereas the other fundamental interactions can be associated to the geometry of the 6 additional ones; in that approach extra fields (which eventually may account for dark matter and/or dark energy) naturally come out by regarding the geometry of the full 10-dimension set.

\section{References}

[1] Landau, L. and Lifshitz, E. (1966) Field Theory. Mir Editions, Moscow.

[2] Weinberg, S. (1972) Gravitation and Cosmology. John Wiley, Hoboken.

[3] Lanczos, C. (1949) Lagrangian Multiplier and Riemannian Spaces. Reviews of Modern Physics, 21, 497. http://dx.doi.org/10.1103/RevModPhys.21.497

[4] Takeno, H. (1964) On the Spin Tensor of Lanczos. Tensor, 15, 103.

[5] Ivanenko, D. and Sardanashvili, G. (1983) The Gauge Treatment of Gravity. Physics Report, 94, 1-45.

[6] Lasenby, A., Doran, C. and Gull, S. (1998) Gauge Theories and Geometric Algebra. Philosophical Transactions of the Royal Society, A356, 487-582. http://dx.doi.org/10.1098/rsta.1998.0178

[7] Blagojevic, M. and Hehl, F.W. (2013) Gauge Theories of Gravitation: A Reader with Commentaries. World Scientific, Singapore. http://dx.doi.org/10.1142/p781

[8] Becchi, C. (1997) Introduction to Gauge Theories. arXiv:hep-ph/9705211v1

[9] Mann, R. (2010) An Introduction to Particle Physics and the Standard Model. CRC Press, Boca Raton.

[10] Roll, P.G., Krotkov, R. and Dicke, R.H. (1964) The Equivalence of Inertial and Passive Gravitational Mass. Annals of Physics, 26, 442-517. 
Scientific Research Publishing (SCIRP) is one of the largest Open Access journal publishers. It is currently publishing more than 200 open access, online, peer-reviewed journals covering a wide range of academic disciplines. SCIRP serves the worldwide academic communities and contributes to the progress and application of science with its publication.

Other selected journals from SCIRP are listed as below. Submit your manuscript to us via either submit@scirp.org or Online Submission Portal.
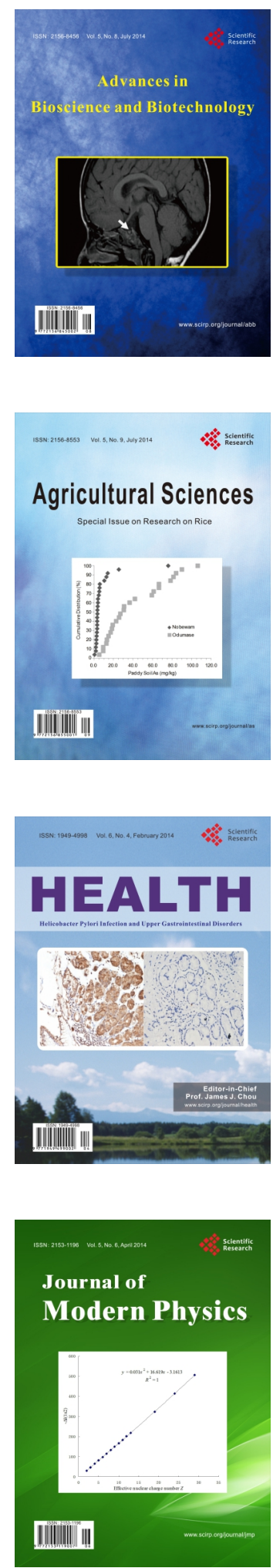
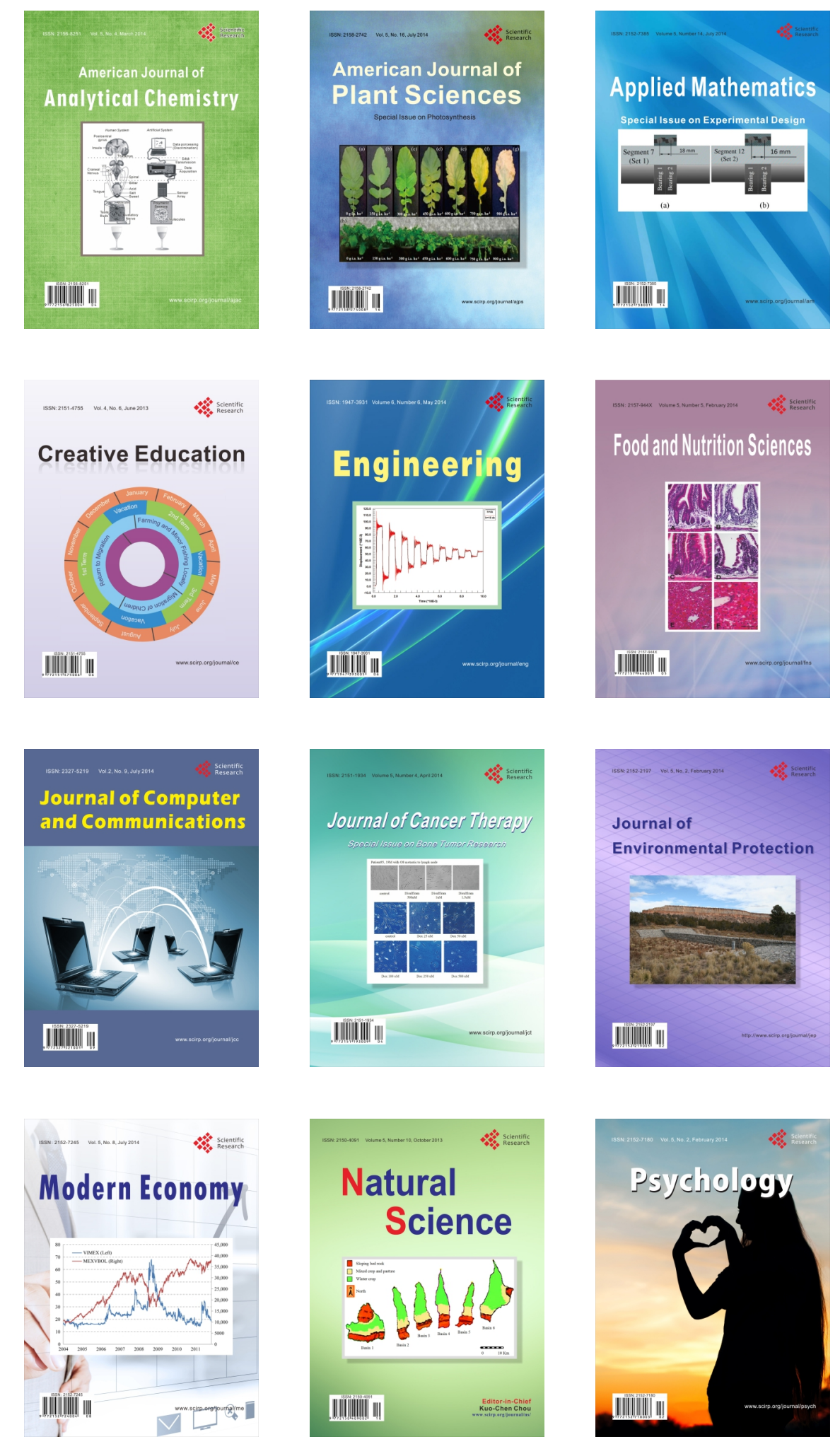